\title{
Biosynthesis of Cellulose by Acetobacter Xylinum. II. Morphological Observations on the Formation of Cellulose Microfibrils by Acetobacter Xylinum
}

\author{
Mitsuo TAKaI and Sadayoshi Watanabe \\ Department of Applied Chemistry, Faculty of Engineering, \\ Hokkaido University, Sapporo, Hokkaido 060, Japan.
}

(Received December 26, 1973)

\begin{abstract}
Electron-microscopic observations of an early stage of a logarithmic phase of bacterial cellulose formation show ill-defined and branched fibrillar elements extending from an amorphous substance of cell envelope. This may be interpreted as the initial crystallization of a cellulosic high polymer. In the photographs of cell cross sections, it has been observed that rather slightly staining rod-like materials about $30-40 \AA$ in breadth and $c a .1000 \AA$ in length are in the cytoplasm of some cells. Composite fibrils become predominant at a middle stage of a logarithmic growth phase. They were in the form of threads having a constant breadth of $c a .500 \AA$ with sharply defined boundaries, and consisting of a few microfibrils. The separate fibrils have intertwined to form tangled masses, and built tough membranes which could not be torn easily. Lysozyme treatment of the microfibril has demonstrated that the shape is a flat ribbon with a constant breadth and a thickness of $c a$. 50-100A. This treatment has the effect of hydrolytic degradation across the axial direction of the microfibril. In addition, a pronounced swelling of the microfibril occurs anisotropically perpendicular to the axis of the microfibril. A bacterial cellulose membrane negatively stained with sodium phosphotungstate, showed a longitudinal periodicity with a $c a .200 \AA$ interval.

KEY WORDS Incubation / Bacterial Cellulose / Fibrillar Element / Cell Envelope / Cross Section / Rod-like Material / Composite Fibril / Microfibril / Tangled Mass / Membrane / Lysozyme Treatment / Negative Staining Periodicity /
\end{abstract}

Electron microscopic studies have shown that cellulose from several different plants has a fibrillar structure $(d=c a$. $100-250 \AA)$. The mechanism for the formation of a uniform cellulose structure is still unknown, and the investigation has been made difficult by the fact that cellulose is produced within the cells of these plants. Therefore, it appears to be more profitable to choose an organism which synthesizes cellulose extracellularly. The original electron microphotographs of Franz and Schiebold ${ }^{1}$ showed that the bacterial membranes consist of a thick network of fibers and ribbons. They concluded that the ribbons had a thickness of about $100 \AA$ and a breadth about $5000 \AA$, and that they were composed of smaller fibers (microfibrils) about $200 \AA$ in diameter. The fact that microfibrils in the walls of plant cells have nearly the same size now raises a question of whether they are indeed primary structural units of cellulose itself. In recent years, high-resolution electron microphotographs ${ }^{2}$ show that a finer unit, which has often been called an "elementary fibril", may exist in Valonia microfibrils treated with ultrasonic waves. A subfilament with a different capacity for absorbing electron dense reagents has also been observed in the crystalline chitin microfibril of the diatom spines. It is similar to the elementary fibril of Valonia. The question is still open as to whether the observed subfilaments are parts of a long, continuous, indigenous elementary fibril of about $35 \AA$ cross-section or whether they are simply portions of the smaller crystallites that have now been revealed directly by more rigorous techniques.

The orientation of a microfibril in a green plant cell wall, and the structure and chemical properties of cellulose have been the subject of 


\section{TAkai and S. Watanabe}

a great deal of study. They are now fairly well understood. In contrast, the mechanism of microfibril formation has received very little attention due principally to the difficulties involved in working with plant material. Two general mechanisms of microfibril formation have been proposed. One is a continued, longitudinal crystallization of the microfibril from a previously formed soluble, polymeric intermediate. ${ }^{3-5}$ The other is a simultaneous polymerization and crystallization of activated glucose residues at a growth tip of an insoluble cellulose microfibril. ${ }^{6}$

Recently we have engaged in a study of the molecular mechanism for the formation of cellulose microfibrils. Even at the present time, there are several problems pending on the structure of cellulose, as followings: whether the chain orientation of the native cellulose microfibril is parallel or antiparallel, whether chain foldings do or do not exist in the cellulose chain, what the staining periodicity reflects from about fine structure, and why a crystallite width calculated from X-ray line breadth differs from that of an electron-microscopic measurement.

\section{MATERIALS AND METHODS}

\section{Method for Quantitative Estimation of Cellulose Synthesis}

Bacterial cells and membranes were prepared as described in Part I. ${ }^{7}$ To esstimate the amount of cellulose formed ${ }^{3} \mathrm{H}$-glucose (specific activity, $9.49 \mathrm{Ci} / \mathrm{mmol}$ ) of $5-\mu \mathrm{Ci} / \mathrm{ml}$ concentration was used with cold glucose as a substrate. The reaction was terminated by $0.5 \mathrm{~N}-\mathrm{NaOH}$. The polymeric alkali-insoluble product was centrifuged at $5000 \mathrm{G}$ for $5 \mathrm{~min}$ and resuspended in $10 \mathrm{ml}$ phosphate buffer solution (PBS; $\mathrm{NaCl} 4 \mathrm{~g}$, $\mathrm{KCl} 0.1 \mathrm{~g}, \mathrm{Na}_{2} \mathrm{HPO}_{4} \cdot 12 \mathrm{H}_{2} \mathrm{O} 1.39 \mathrm{~g}, \mathrm{KH}_{2} \mathrm{PO}_{4} 0.1 \mathrm{~g}$ per $500 \mathrm{ml}$ of distilled water; $\mathrm{pH}$ 7.2). The same operation was repeated five times to remove as much of the soluble ${ }^{3} \mathrm{H}$ as possible. In the last stage, the insoluble product was resuspended in $1-\mathrm{m} l \mathrm{PBS}$ and an equal volume of $4-\% \mathrm{NaOH}$ added. The solution was filtered on glass fiber paper (Whatmen GF/C) and washed with 2-\% $\mathrm{NaOH}$ and water three times, respectively. The filtrates were dried with the glass-fiber paper at $80^{\circ} \mathrm{C}$ for $24 \mathrm{hr}$. Their radioactivities were counted in a solution scintillator of toluene, dimethylPOPOP and 2,5-diphenyl-oxazole (PPO) by a scintillation counter (Isotope Center of Hokkaido University, Sapporo).

\section{Preparation of Specimens for Electron Microscopy}

Whole cells were prepared for electron microscopy by using 1-\% osmium tetroxide to fix a pellet of the cells obtained by centrifugation. The fixed, stained cells were dehydrated step wise with ethanol and acetone, and embedded in Epock 533-MNA-DMP $30(13: 11: 0.5)$ by Kushida's method. Then they were polymerized in an electric oven at $50^{\circ} \mathrm{C}$ for $50 \mathrm{hr}$ with $a \# 0$ gelatine capsul and sectioned with a LKB model 4801A ultramicrotome to give an interference color of silver gray to gold. After cementing the section onto the copper grids by mesh cement, the specimens were further stained with uranyl acetate and lead acetate by a variation of the method of Watson ${ }^{8}$ and Reynolds. ${ }^{9}$

In order to observe the elongation process of cellulose microfibrils, specimens were prepared by the following procedure. One volume of standard cell suspension (about $10^{9}$ viable cells per $\mathrm{m} l$ ) was filtered through gossypium cotton, and then added to 1 volume of $2-\%$ glucose and $0.5 \%$ yeast extract. The mixture was incubated with gentle shaking for $30 \mathrm{~min}$, after which growth was stopped by adding a half volume of glacial acetic acid. The aqueous suspension was then diluted with 35 volumes of phosphate buffer solution. One $\mathrm{ml}$ of the suspension was further diluted with $35 \mathrm{~m} l$ of water. Drops of this diluted suspension were placed on the grids with collodion films thinly coated with carbon. They were washed three times by flotation over distilled water kept on the parafilm and the water removed by absorbing it with a strip of filter paper. After drying, the material on the grids was either shadowed with $\mathrm{Pt}-\mathrm{C}$ or negatively stained with sodium phosphotungstate by the method of Pease. ${ }^{10}$ Sodium phosphotungstate was placed on the material on the grids by pipette and stained for $5 \mathrm{~min}$, the excess removed with a strip of filter paper and the sample washed with water in the same manner as mentioned above. All specimens were photographed using Hitachi model HS-8 and HS-11-DS electron microscopes. 
Biosynthesis of Cellulose by Acetobacter Xylinum. II.

Preparation of Specimens for Scanning Electron Microscopy

A large section of cellulose membrane was dried with the flat surface resting on a horizontal glass plate. The dried sheet was peeled off and used for scanning electron-microscopic observation. These specimens were mounted on specimen stubs with silver paste and coated with gold in a vacuum of $5 \times 10^{-5}$ torr. All specimens were photographed using JEOL JSM-1 and JSM-2 models of scanning electron microscope. The specimen stub was inclined at $45^{\circ}$ from the horizontal and a $25-\mathrm{kV}$ accelerating voltage was used to examine the specimen surfaces.

\section{RESULTS AND DISCUSSION}

\section{Cellulose Synthesis in Microscale Culture}

The incorporation curves of ${ }^{3} \mathrm{H}$-glucose into cellulose consisted of the next three phases with respect to incubation time. (1) a lag phase, (2) a logarithmic phase, and (3) a declining phase (Figure 1).

The lag phase at short incubation times (less than $15 \mathrm{~min}$ ) will be due to a time lag of the

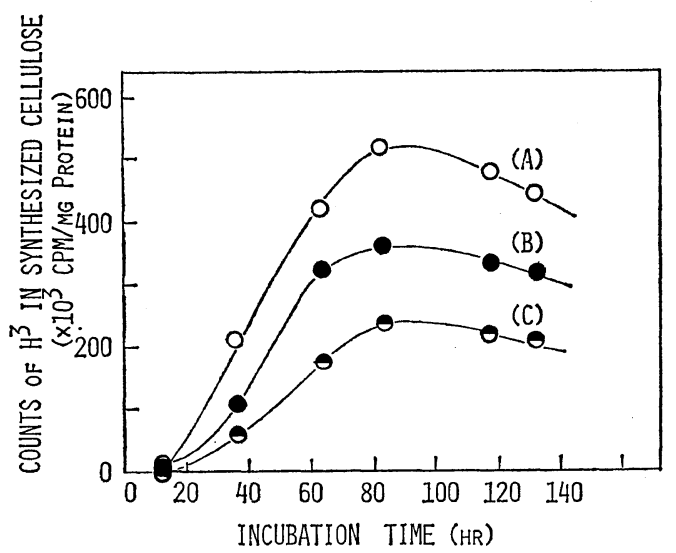

Figure 1. Synthesis of cellulose from ${ }^{3} \mathrm{H}$-glucose as a function of incubation time. Suspentions of whole cells free from cellulose and containing approximately $10^{9}$ viable cells per $\mathrm{m} l$ in sterilized substrate solution were prepared at first. $0.1 \mathrm{~m} l$ of them and ${ }^{3} \mathrm{H}$-glucose (specific activity, $9.49 \mathrm{Ci} /$ $\mathrm{mmol}$ ) of $5 \mu \mathrm{Ci} / \mathrm{m} l$ concentration were put into $0.8-\mathrm{m} l$ liquid medium. Cellulose was estimated in $\mathrm{CPM} / \mathrm{mg}$ Protein as described above Materials and Methods. Cold glucose concentrations in the medium are (A) $0.5 \%$, (B) $1 \%$, and (C) $2 \%$. cellulose production after inoculation. Another possibility is that an alkali-soluble polymer is produced in the phase, because the higher a cell concentration, the shorter the period of the lag phase. The logarithmic phase, the fastest rate of incorporation of ${ }^{3} \mathrm{H}$-glucose into cellulose, is between 30 and $60 \mathrm{~min}$ of incubation regardless of the given glucose concentration. The incorporation of ${ }^{3} \mathrm{H}$-glucose into cellulose reached a maximum after $80 \mathrm{hr}$ and then slowly decreased. The protein content in the supernatant of detergent showed a maximum after $80 \mathrm{hr}$, too (Figure 2 ). It is common knowledge that cellulose microfibrils are degraded in a variety of biological

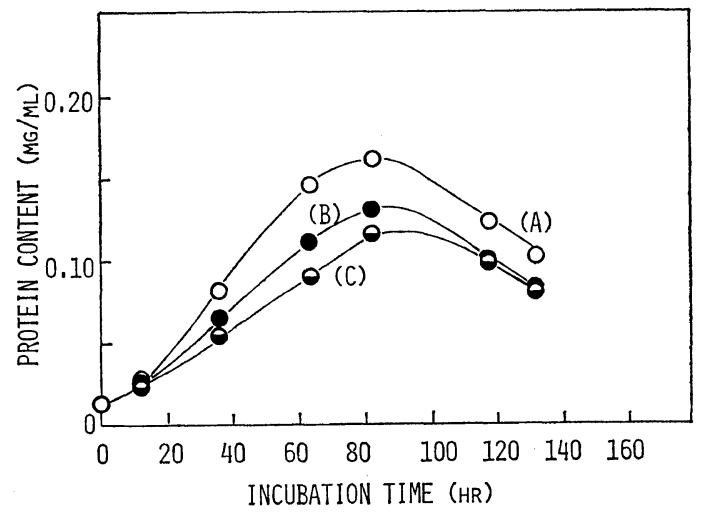

Figure 2. Decreasing effect of prolonged incubation on the protein content in the supernatant of detergent measured by optical density at $280 \mathrm{~m} \mu$. Cold glucose concentrations in the medium are (A) $0.5 \%$, (B) $1 \%$, and (C) $2 \%$.

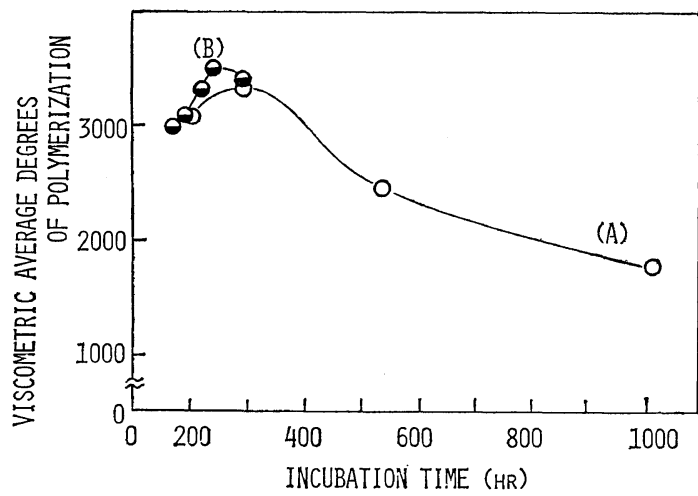

Figure 3. Decreasing effect of prolonged incubation on the degree of polymerization of bacterial cellulose: (A) static culture; (B) static culture at first for three days and then shaken for each duration. 
processes. The newly formed cellulose microfibrils are quickly attacked and degraded by cellulase released into the medium at the same tim. $\overline{\mathrm{DP}}$ of products attained a maximum value of 3500 after $250 \mathrm{hr}$ and then also decreased (Figure 3). The decreasing $\overline{\mathrm{DP}}$ indicates the action of cellulase.

On the other hand, in the large scale culture it was found that the yield of cellulose was as high as $4 \%$ on the basis of glucose weight in the system of combined static and shaken cultures.

Identification and General Morphology of. Extracellular Polymer

Photographs of cross-sections of fixed and stained cells of Acetobacter Xylinum in the lag phase are shown in Figure 4. These cells were presumably engaged in active cellulose synthesis at the time of fixation. In general, the structure of the cell envelope and its contents are similar to those found in gram-negative bacteria. ${ }^{11}$ The cell wall consists of three layers, the outer and inner layers staining more deeply than the middle layer. The dimension of these layers is about $200 \AA$ and corresponds, in general, to Robertson's model $^{12}$ for the basic structure of biomembranes. In the cytoplasm of some cells, there are rod-like materials with $30-40 \AA$ in breadth and $1000 \AA$ in length, slightly staining with uranyl and lead acetates. At present it is not apparent whether these materials are associated with the biosynthesis of cellulose or not. Electron-microscopic observations of an early stage of a logarithmic phase of bacterial cellulose formation show illdefined and branched fibrillar elements extending from the amorphous surface of the cell envelope (Figure 5). It has been speculated that this fibrillar element might be extracellular and soluble proteins contained in the cultures of Acetobacter Xylinum. When dried on the electronmicroscope grids, these proteins may easily form ill-defined filaments that might easily be mistaken for the beginning of microfibril. Therefore specimens for electron microscopy were thoroughly washed with saline, phosphate buffer, and distilled water. Since the fibrillar elements were insoluble in $1-\% \mathrm{NaOH}$, it seems that they may be cellulosic materials as the beginning of crystallization of a cellulosic high polymer.

Cellulose fibers have begun to form rapidly after the early stage of the logarithmic phase. As can be seen from Figure 6, the growth of cellulose fibril has not taken place within a bacterium or from its surface, but rather at some distance from the organism. Composite fibrils became predominant in the fields at a middle stage in the logarithmic phase. The composite

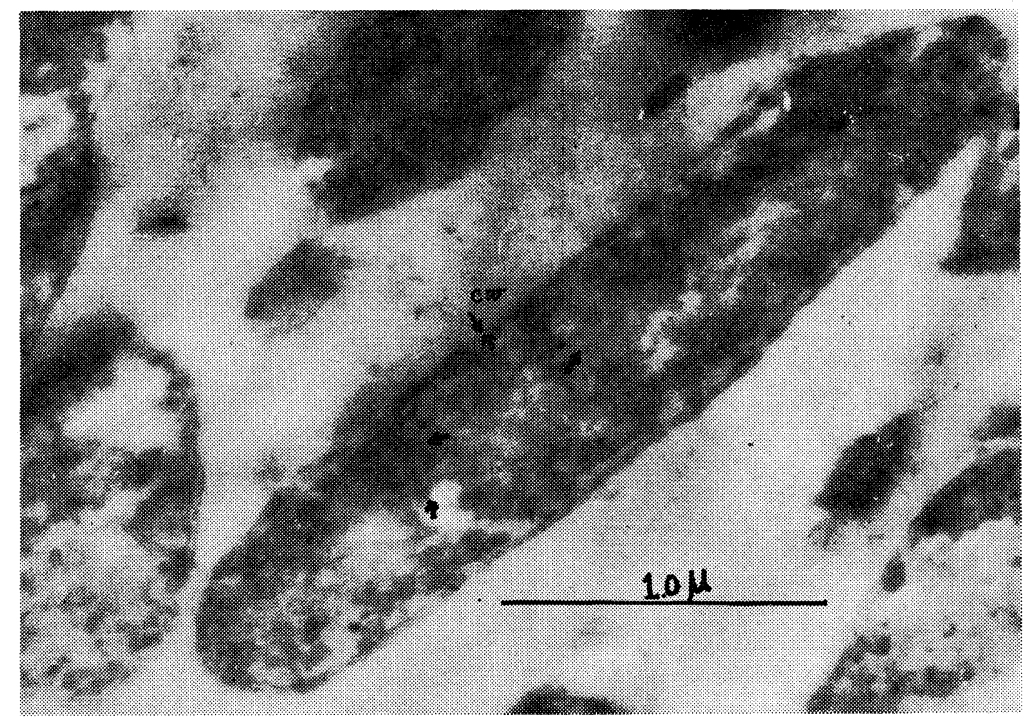

Figure 4. Electron microphotograph of a section of Acetobacter Xylinum cell showing the membrane structure of cell envelope and rod-like materials (indicated by arrows) in a cytoplasm, $\mathrm{CW}$, cell wall. 
Biosynthesis of Cellulose by Acetobacter Xylinum. II.

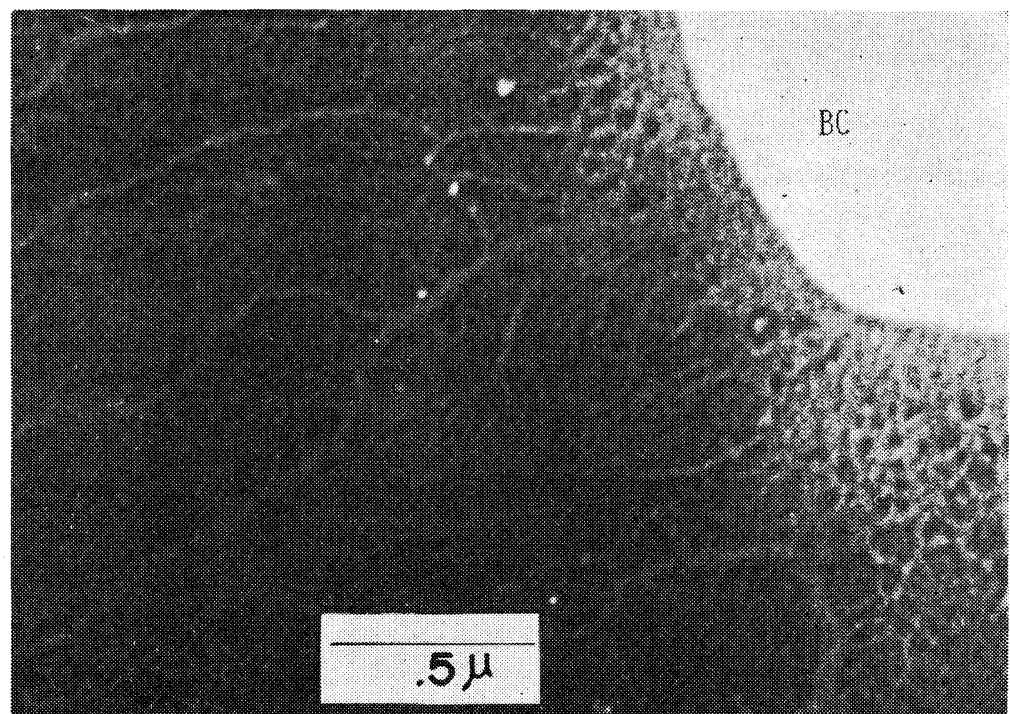

Figure 5. Electron microphotograph of branched fibrillar elements extending from amorphous surface of the cell envelope at an early stage of bacterial cellulose formation, BC, bacteria cell.

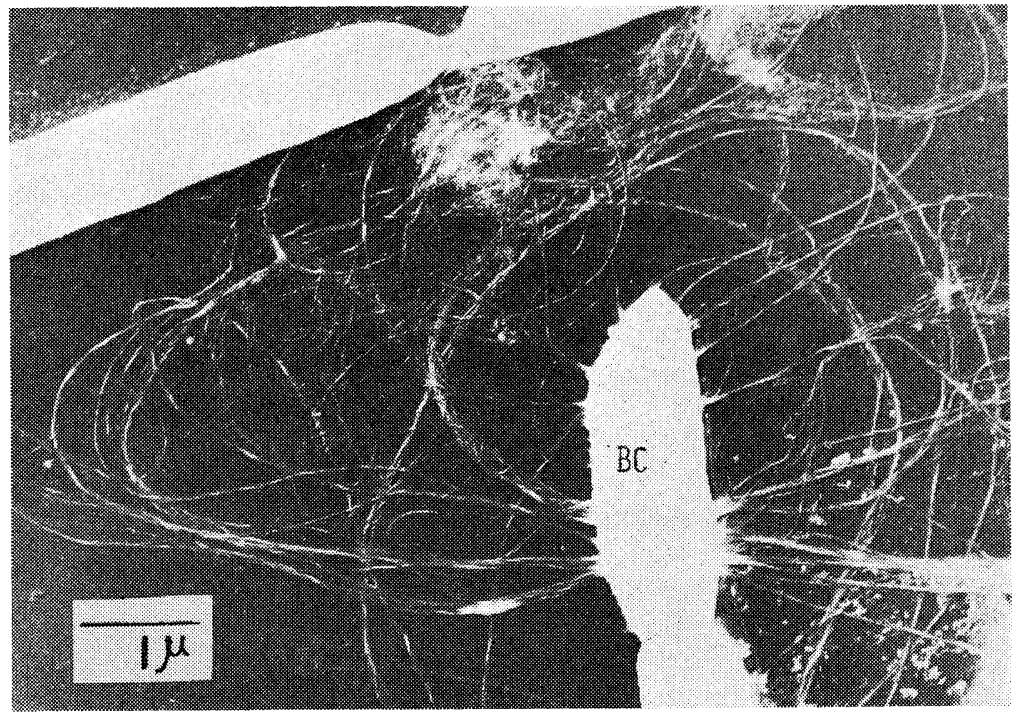

Figure 6. Electron microphotograph of composite fibrils at a middle stage of bacterial cellulose formation. The separate microfibrils have intertwined to form tangled masses, BC, bacteria cell.

fibrils are in the form of threads with a constant breadth of $c a$. $500 \AA$ and sharply defined boundaries, and consist of a few microfibrils. As to the dimensions of the subelements of cellulose, it has been argued that the observed width of microfibril does not coincide with the crystallite width determined by $\mathrm{X}$-ray diffraction, and whether or not the fibril with a width of $35 \AA$, the "elementary fibril", is the indigenous morphological element. Much electron-microscopic evidence for a $35-\AA$ elementary fibril has been obtained for a wide variety of cellulosic substances. Evidence of large structures is then suggested as resulting from a lateral coherence of 


\section{TAKAI and S. WATANABE}

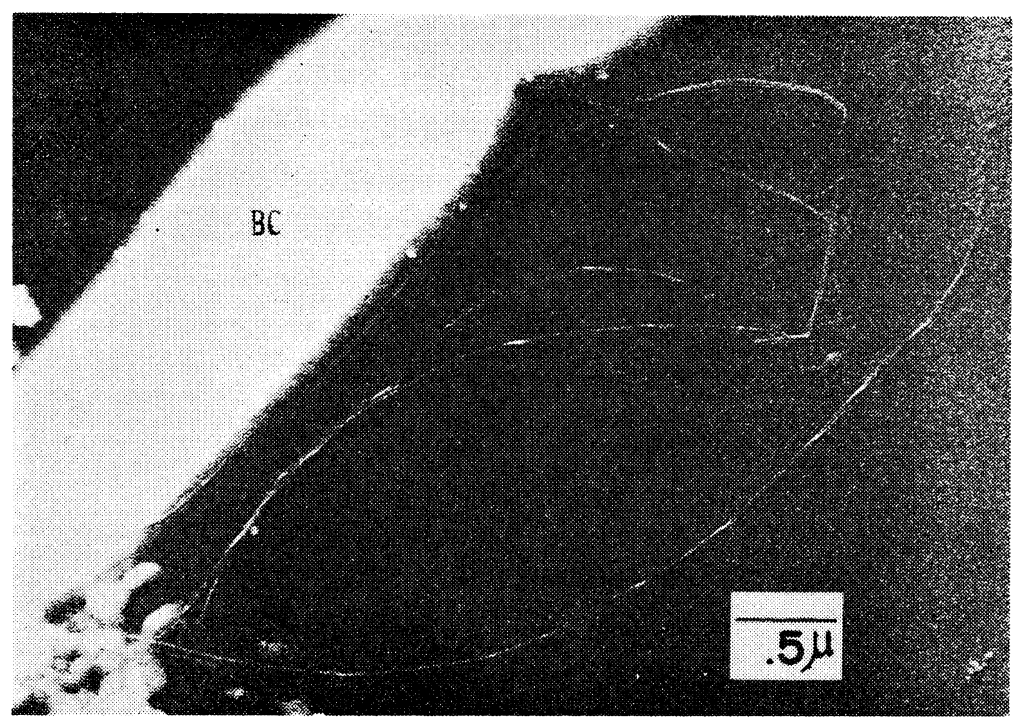

Figure 7. A typical electron microphotograph of twisting in composite cellulose fibrils. Broadening of the fibril image suggests the ribbon-shape structure, BC, bacteria cell.

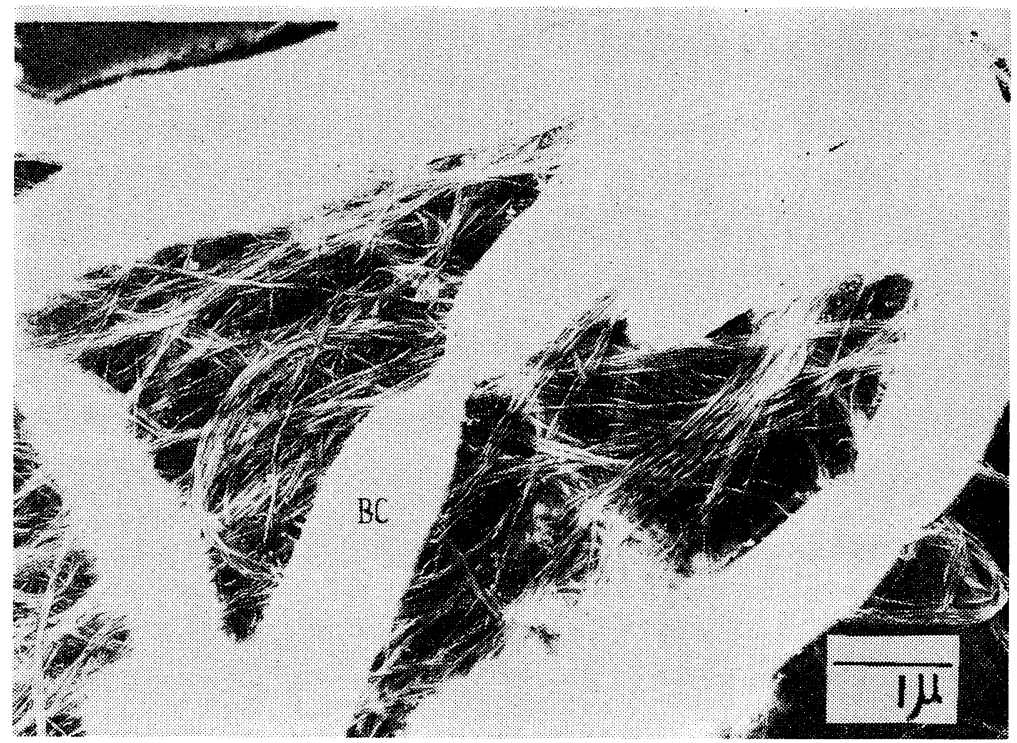

Figure 8. Tangled cellulose microfibrils in an old culture. Cells formed a mat of microfibrils in which they are embedded, BC, bacteria cell.

a number of elementary fibrils. This coherence is inferred to occur during the drying process. The mechanism for this type of coherence, whereby the paracrystalline surfaces of the smaller units become incorporated into a perfected crystal, must then be explained. A twisting of a portion of the microfibril was observed for each $0.5-1 \mu$ along the fibrillar axis (Figure 7). This may be interpreted as a typical Eshelby twist ${ }^{13}$ due to the uncompensated torque induced by the anisotropic shrinkage in the flat ribbon-like microfibril during the free drying process. They give the same appearance as many metallic or inorganic "whiskers". The separate fibrils have interwined to form a tangled mass (Figure 8). Cells which metabolized glucose for two days formed a mat 
Biosynthesis of Cellulose by Acetobacter Xylinum. II.

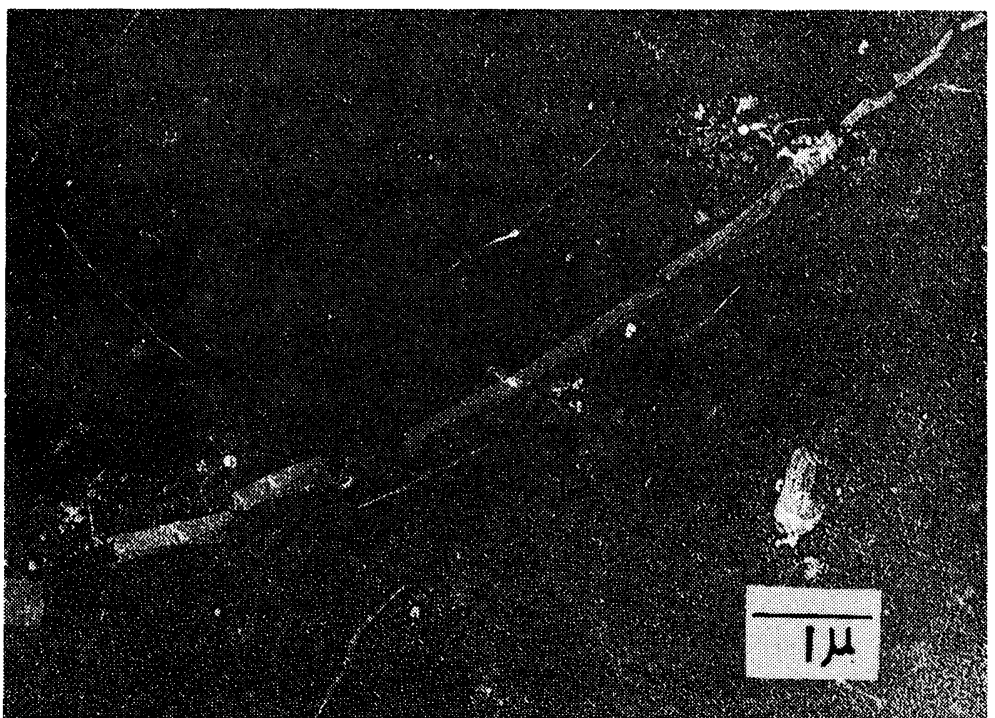

Figure 9. Electron microphotograph of microfibrils treated with lysozyme of $800 \mu \mathrm{g} / \mathrm{m} l$ at $30^{\circ} \mathrm{C}$ for $1 \mathrm{hr}$. The treatment made more clear the ribbon-shape of microfibrils.

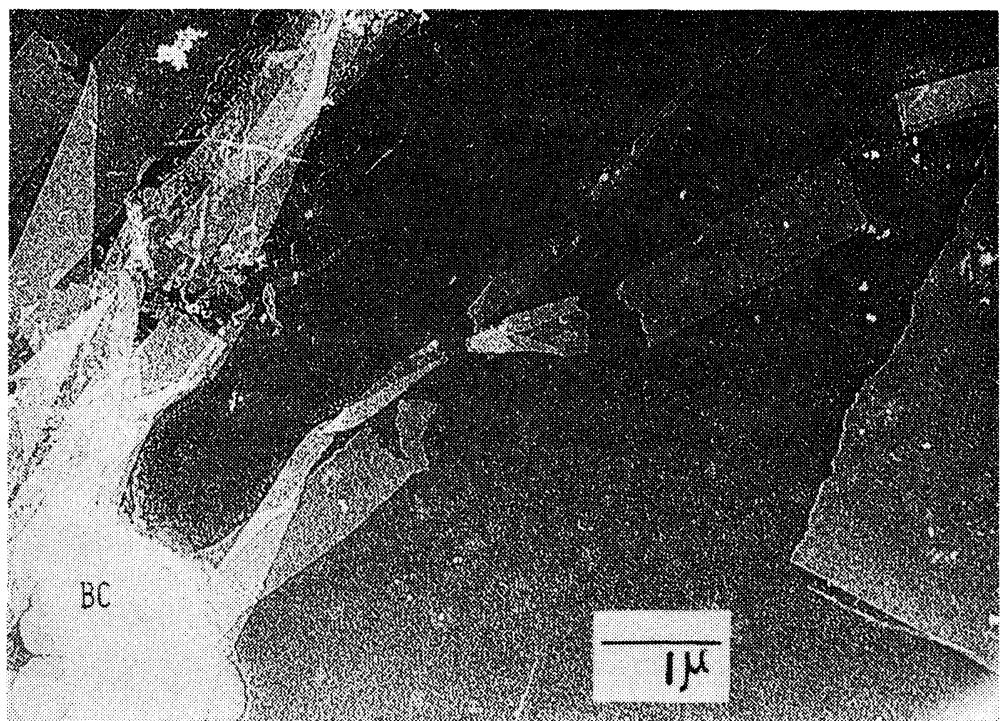

Figure 10. Electron microphotograph of large membranous substances observed after the lysozyme treatment of cellulose microfibrils, $\mathrm{BC}$, bacteria cell.

of cellulose microfibrils in which the cells are embedded. It was a tought membrane which could not be torn easily.

Morphology of Cell and Microfibril Treated with Lysozyme

In a various incubation time, lysozyme was added to the cultures $(800 \mu \mathrm{g} / \mathrm{m} l)$ and with occasional shaking incubated for $1 \mathrm{hr}$ at $30^{\circ} \mathrm{C}$.
With the lysozyme treatment it was found that the shape of a microfibrils a flat ribbon with a

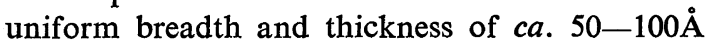
(Figure 9). Furthermore this treatment gave the effect of a degradational hydrolysis of the microfibril perpendicular to its axis. In addition, a pronounced swelling of the microfibrils occurs anisotropically across the width of the microfibril. 
As reported by Tønnensen and Ellefesen, ${ }^{14}$ this could be explained by unrolling the microfibril bundles composed of rolling sheets with a selective uniplanar orientation of the (101) plane. However, it is nothing more than speculation because the effect of lysozyme on the cellulose structure, especially, the hydrogen bonding within the rolling sheets, is unknown. In another photograph with a large scale in $\mu$ units (Figure 10), a membraneous substance was also observed. It is not apparent as yet whether this arose from cellulose microfibrils or cell membranes.

\section{Scanning Electron Microphotographs}

The scanning electron microscopy (SEM) is essentially different from a conventional electron microscopy in observing images by the reflected secondary electron from the specimen surface coated with $\mathrm{Au}$ metal. A face view of the membrane of bacterial cellulose is given in Figure 11. As shown in the photograph, the shape of the cellulose microfibril is continuous and long having a breadth of $c a .200 \AA$. The arrangement of microfibrils is irregular, but the breadth remains constant over a long distance. However, the internal structure of the microfibrils cannot be interpreted from this photograph. Figure 12 shows a SEM photograph of microfibrils of Valonia macrophysa cellulose membrane, with a breadth of about $250 \AA$. The outer lamellars show many superposed and rather distinct layers

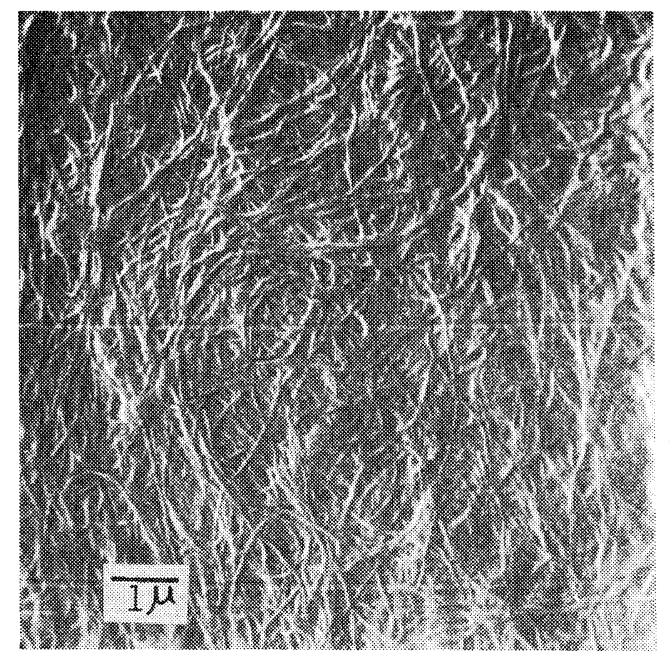

Figure 11. Scanning electron microphotograph of bacterial cellulose membrane. in the wall, and it might therefore be expected that the direction of the cellulose microfibrils and chains would alternate from layer to layer. The thinnest layer which can be stripped and be mounted in an X-ray spectrometer gives a crossed photograph of typical Valonia. This is equivalent to that of two sets of ramie fibers crossed at about $90^{\circ}$ to each other. In other words, the wall has not one but two sets of cellulose chains making about a $90^{\circ}$ angle with each other. Figure 13 shows a SEM photograph of a ramie single fibers. The breadth of microfibrils is the

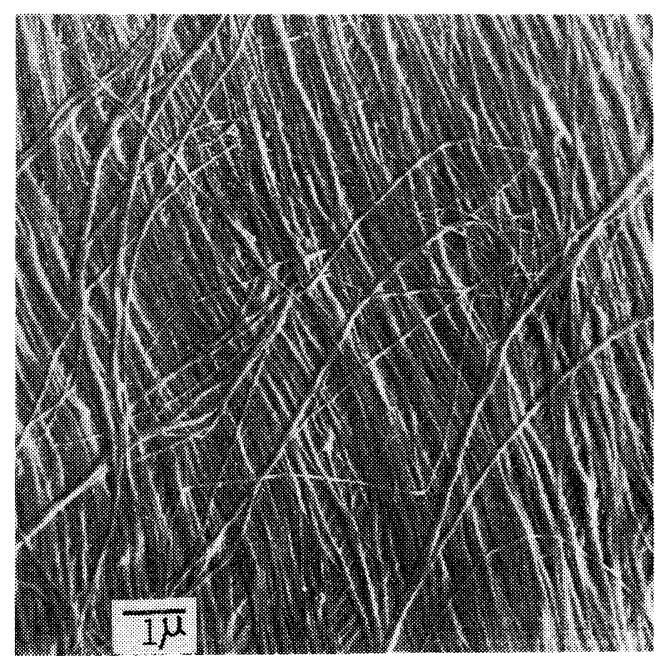

Figure 12. Scanning electron microphotograph of Valonia cellulose membrane.

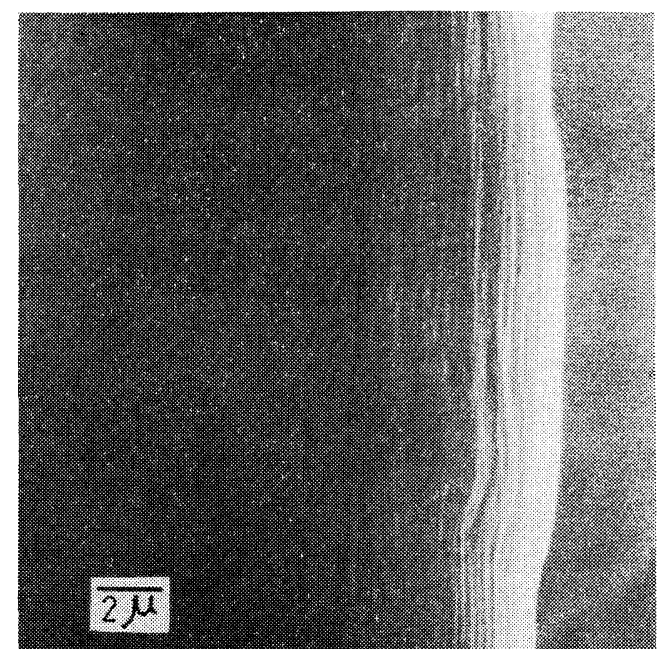

Figure 13. Scanning electron microphotograph of ramie cellulose monofilament. 


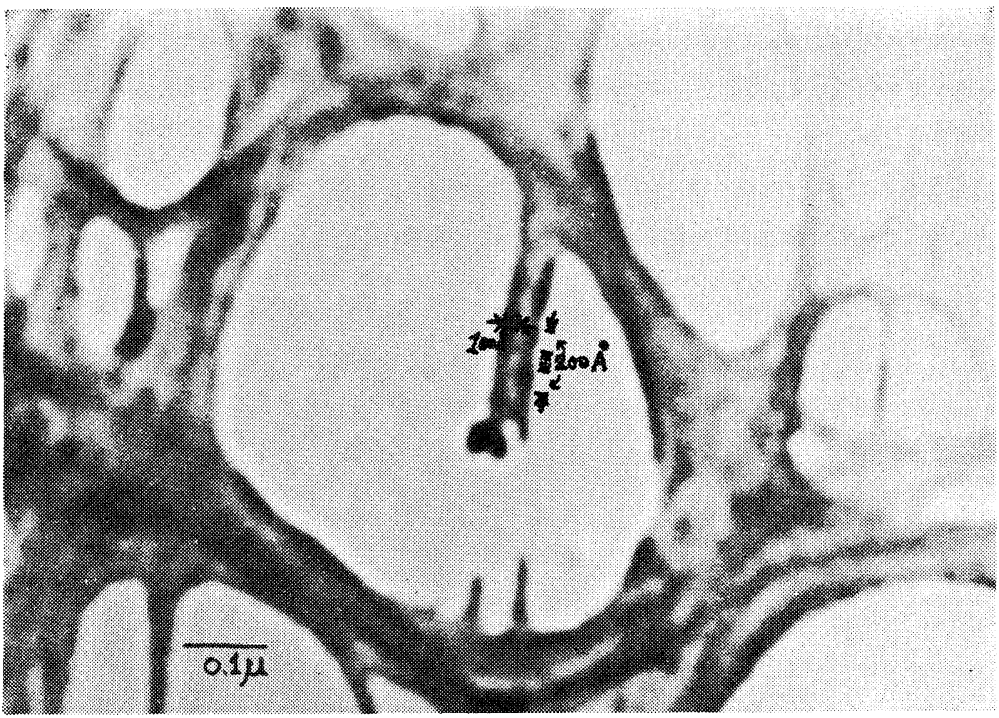

Figure 14. Electron microphotograph of bacterial cellulose microfibrils negatively stained with sodium phosphotungstate. Longitudinal periodicity ( $c a .200 \AA$ interval) along the fibrillar axes can be seen.

same order of magnitude as bacterial and Valonia cellulose. The arrangement of them is straight in the fiber axis. However, the spiral angles against the fiber axis could not be measured from this photograph.

Negative Staining Periodicity of Microfibril with Sodium Phosphotungstate

Figure 14 shows and electron-microscopic observation of a bacterial cellulose membrane stained with sodium phosphotungstate. The longitudinal periodicity appears with a $c a .200-\AA$ interval period along the cellulose microfibril. A similar periodicity was long known in natural fibers such as collagen and keratin, and was primarily considered to be a consequence of the growth process. No long period interference was observed in small angle X-ray scattering patterns of the bacterial cellulose before and after hydrolysis with hydrochloric acid. However, the regenerated cellulose prehydrolyzed with the acid gave a small angle interference. It is a meridonal interference corresponding to a interlattice plane distance of $c a$. $200 \AA$. The result is consistent with that of the electron-microscopic study. In regenerated cellulose, therefore, this may reflect some periodic process that in itself may be related to the structure of the microfibril. On the other hand, in native cellulose, this periodicity phenomena are unusual. Thus it was recognized that this periodicity might be an artifact, but if not, it was proposed that it may reflect that the microfibril is composed of a flat ribbon wound in the form of the tight helix described by Manley. ${ }^{15}$

Acknowledgment. This investigation had been supported in part by a grant aid for scientific research from the Japanese Government in 1972 and 1973.

The authors are grateful to Mr. Y. Shiono for advice in preparing the sections and taking the electron microphotographs.

\section{REFERENCES}

1. E. Franz and E. Schiebold, J. Makromol. Chem., 1, 4 (1943).

2. K. H. Gardner and J. Blackwell, J. Polym. Sci., Part C, 36, 327 (1972).

3. S. Hestrin and M. Schramm, Biochem. J. (London), 58, 345 (1954).

4. I. Ohad, D. Danon, and S. Hestrin, J. Cell Biol., 12, 31 (1962).

5. G. Ben-Hayyim and I. Ohad, ibid., Part 2, 25, 191 (1965).

6. J. R. Colvin, "The Formation of Wood in Forest Trees", M. H. Zimmerman Ed., Academic Press, New York, N. Y., 1964, p 189. 


\section{TAKaI and S. WATANABE}

7. M. Takai and S. Watanabe, Polymer J. in contribution.

8. M. L. Watson, J. Biophys. Biochem. Cytol., 4, 475 (1958).

9. E. S. Reynolds, J. Cell Biol., 17, 208 (1963).

10. D. C. Pease, "Histological Techniques for Electron Microscopy', Academic Press, New York, N. Y., 1964, p 236.

11. E. Kellenberger and A. Ryter, J. Biophys. Biochem. Cytol., 4, 323 (1958).
12. J. D. Robertson, J. Biophys. Biochem. Cytol., 3, 1043 (1957).

13. J. D. Eshelby, J. Appl. Phys., 24, 176 (1953).

14. B. A. Tønnesen and $\emptyset$. Ellefsen, "Cellulose and Cellulose Derivatives", N. M. Bikales and L. Segal Ed., John Wiley \& Sons, Inc.-Interscience, New York, N. Y., Part IV, Chapter XIII F, 1971, p 265.

15. R. St. J. Manley, Nature, 204, 1155 (1964). 